\title{
Analyzing the Impacts of Natural Environments on Launch and Landing Availability for NASA’s Exploration Systems Development Programs
}

\author{
Karen M. Altino ${ }^{1}$ \\ NASA Marshall Space Flight Center, Huntsville, AL 35812 \\ K. Lee Burns, Ph.D. ${ }^{2}$ \\ Raytheon/Jacobs ESSSA Group, Huntsville, AL 35806 \\ Robert E. Barbré, Jr. ${ }^{3}$ \\ Jacobs/Jacobs ESSSA Group, Huntsville, AL 35806 \\ and \\ Frank B. Leahy ${ }^{4}$ \\ NASA Marshall Space Flight Center, Huntsville, AL 35812
}

The National Aeronautics and Space Administration (NASA) is developing new capabilities for human and scientific exploration beyond Earth orbit. Natural environments information is an important asset for NASA's development of the next generation space transportation system as part of the Exploration Systems Development (ESD) Programs, which includes the Space Launch System (SLS) and Multi-Purpose Crew Vehicle (MPCV) Programs. Natural terrestrial environment conditions - such as wind, lightning and sea states - can affect vehicle safety and performance during multiple mission phases ranging from pre-launch ground processing to landing and recovery operations, including all potential abort scenarios. Space vehicles are particularly sensitive to these environments during the launch/ascent and the entry/landing phases of mission operations. The Marshall Space Flight Center (MSFC) Natural Environments Branch provides engineering design support for NASA space vehicle projects and programs by providing design engineers and mission planners with natural environments definitions as well as performing custom analyses to help characterize the impacts the natural environment may have on vehicle performance. One such analysis involves assessing the impact of natural environments to operational availability. Climatological time series of operational surface weather observations are used to calculate probabilities of meeting/exceeding various sets of hypothetical vehicle-specific parametric constraint thresholds. Outputs are tabulated by month and hour of day to show both seasonal and diurnal variation. This paper will discuss how climate analyses are performed by the MSFC Natural Environments Branch to support the ESD Launch Availability (LA) Technical Performance Measure (TPM), the SLS Launch Availability due to Natural Environments TPM, and several MPCV (Orion) launch and landing availability analyses - including the 2014 Orion Exploration Flight Test 1 (EFT-1) mission.

\footnotetext{
${ }^{1}$ Flight Vehicle Atmospheric Environments, Natural Environments Branch, NASA/MSFC EV44, Member.

${ }^{2}$ Sr. Physics Engineer II, Natural Environments Branch, NASA/MSFC EV44, Non-Member.

${ }^{3}$ Natural Environments Engineer, Natural Environments Branch, NASA/MSFC EV44, Non-Member.

${ }^{4}$ Flight Vehicle Atmospheric Environments, Natural Environments Branch, NASA/MSFC EV44, Non-Member.
} 


\section{Nomenclature}

$\begin{array}{ll}A O A & =\text { Abort-Once-Around } \\ A P R A & =\text { Atmospheric Parametric Risk Assessment } \\ C M & =\text { Crew Module } \\ C x P & =\text { Constellation Program } \\ D R M & =\text { Design Mission Reference } \\ E F T-1 & =\text { Exploration Flight Test } 1 \\ E R A-40 & =40 \text {-year European Centre for Medium Range Weather Forecasts Re-Analysis } \\ E R A-I & =\text { Interim European Centre for Medium Range Weather Forecasts Re-Analysis } \\ E S D & =\text { Exploration Systems Development } \\ G U I & =\text { graphical user interface } \\ K S C & =\text { Kennedy Space Center } \\ k m & =\text { kilometer } \\ L A & =\text { launch availability } \\ L A S & =\text { Launch Abort System } \\ L C-39 B & =\text { Launch Complex 39B } \\ L E O & =\text { low Earth orbit } \\ m & =\text { meter } \\ M A P & =\text { Mission Analysis Package } \\ M P C V & =\text { Multi-Purpose Crew Vehicle } \\ M S F C & =\text { Marshall Space Flight Center } \\ N A S A & =\text { National Aeronautics and Space Administration } \\ N E C & =\text { non-exceedance condition } \\ N L D N & =\text { National Lightning Detection Network } \\ P_{C A} & =\text { climatological availability probability } \\ P_{F M} & =\text { failure mode probability } \\ P_{V} & =\text { probability of violation } \\ P A C E R & =\text { Probabilities of Atmospheric Conditions and Environmental Risk } \\ P O R & =\text { period of record } \\ S L F & =\text { Shuttle Landing Facility } \\ S L S & =\text { Space Launch System } \\ S M & =\text { Service Module } \\ S S P & =\text { Space Shuttle Program } \\ T P M & =\text { Technical Performance Measure } \\ U T C & =\text { Coordinated Universal Time }\end{array}$

\section{Introduction}

$\mathrm{N}$ atural environments need to be considered for the design and operation of aerospace vehicles. Spacecraft must be able to survive the environmental conditions encountered during each phase of a mission timeline. For space launch vehicles, design engineers and mission planners need to understand launch site weather in order to develop robust vehicle designs and operational concepts that will allow high launch probabilities. In addition to vehiclespecific environmental constraints, range safety constraints must also be considered. The MSFC Natural Environments Branch has the ability to perform climate analyses for potential launch and landing sites. For the ESD LA TPM, two different scenarios need to be considered - SLS cargo-only missions, and SLS-Orion missions. Both configurations will launch from the Kennedy Space Center (KSC) in Florida, therefore KSC pad/near-pad weather conditions need be assessed. Range safety constraints also need to be considered for both mission types. However, for the SLS-Orion configuration, sea states along the Atlantic Ocean ascent abort track and the Pacific Ocean AbortOnce-Around (AOA) splashdown zone need to be considered so that the crew can both survive and be retrieved by recovery forces in a timely manner.

The MSFC Natural Environments Branch has a long history of computing mission phase availability with respect to natural environmental conditions. During the Apollo program, a series of analysis tools - known collectively as the Mission Analysis Package (MAP) - were developed and used successfully for mission planning purposes. During the Space Shuttle Program (SSP), these various software programs were combined with additional features and capabilities resulting in the Atmospheric Parametric Risk Assessment (APRA) statistical analysis tool. 
Both MAP and APRA were primarily designed to analyze launch availabilities. When NASA's Constellation Program (CxP) began, additional emphasis was placed on conditions at landing sites as well as potential oceanic abort locations. Rather than adding new functionality to APRA, it was determined to be more effective to create a new availability model. The new software package was developed and referred to as Probabilities of Atmospheric Conditions and Environmental Risk (PACER) ${ }^{1}$. This tool is currently being used to assess scenarios for ESD, SLS, and MPCV.

\section{PACER Climate Analysis Tool}

Functionally, PACER consists of three distinct program components: (1) a set of offline utility routines for creating and formatting input datasets, (2) a graphical user interface (GUI) containing various keyboard and mouseactivated widget controls used to define an analysis scenario, and (3) a computational engine for calculating the output probabilities. The PACER methodology is straightforward. The sequential processes involved are detailed below.

\section{A. Identifying Environmental Parameters and Geographical Sites of Interest}

Environmental parameters can be any surface-based observable quantity. Currently implemented parameters are shown in Table 1. Geographical sites can be any surface location where a vehicle is expected to operate and for which data is available. These can include launch sites such as KSC as well as oceanic landing zones and potential ascent abort ground tracks. Defining these parameters and sites is performed during software generation, as the parameter and site lists are hard-wired into the code. However, PACER has a modular implementation that allows rapid integration of new parameters and sites once interest and data become available.

Table 1. Currently implemented PACER environmental parameters.

\begin{tabular}{|c|}
\hline ENVIRONMENTAL PARAMETERS \\
\hline Air Temperature \\
Dewpoint Temperature \\
Land-based Mean Wind Speed \\
Land-based Peak Wind Speed \\
Visibility \\
Cloud Ceiling Height \\
Sky Cover \\
Presence of Precipitation \\
Presence of Thunderstorms \\
Distance to Nearest Lightning Strike \\
Oceanic Mean Wind Speed \\
Significant Wave Height \\
Average Wave Period
\end{tabular}

\section{B. Generating Time Series Datasets for All Parameters/Sites of Interest}

Ideally, each time series dataset will have hourly observations throughout the global period of record (POR) that runs from January 1, 1975 through the current day. For practical reasons, the current day is reset and each dataset is updated at periodic intervals. Presently, the global POR extends through December 31, 2012. It should be noted that each parameter dataset will have its own specific POR and reporting cycle. For example, significant wave heights are only available every six hours. Likewise, lightning distance observations are not available prior to 1988. Most of the time series datasets also have occasional intervals where observations are not available due to instrumentation 
maintenance, etc. Dataset generation is performed prior to any specific PACER analysis session using the offline utility routines.

\section{Defining a Specific PACER Analysis Scenario}

A scenario definition consists of a selected set of parameters to be analyzed along with constraint threshold criteria. Each parameter/constraint specification is referred to as a non-exceedance condition (NEC). For example, one could choose to analyze visibility and set a threshold value of 1 kilometer $(\mathrm{km})$. Then the corresponding NEC would be that a reported visibility was greater than $1 \mathrm{~km}$. This process is performed manually using GUI controls.

\section{Submitting the Scenario Definition to the Computational Engine to Calculate Resultant Probabilities}

Calculations are performed in the background and tabular results are saved to text files. The values of various environmental parameters are not statistically independent. For example, the presence of thunderstorms is generally associated with lower cloud ceiling heights compared to clear days. However, the exact relationship between pairs of variables is stochastic and cannot be treated deterministically. Thus, an integrated approach is required to determine the effect of environmental conditions on overall mission availabilities.

Functionally, PACER first determines the number of reporting intervals with valid observations for all parameters within the overlapping PORs for all datasets used for a given analysis. For each valid reporting interval, each parameter value is compared to its corresponding constraint threshold. If one or more parameter NEC is violated, that reporting interval is flagged as a "fail". PACER then computes the probability of violation $\left(\mathrm{P}_{\mathrm{V}}\right)$ as the ratio of the number of failing reporting intervals divided by the total number of valid intervals. The climatological availability probability $\left(\mathrm{P}_{\mathrm{CA}}\right)$ is the value of $\mathrm{P}_{\mathrm{V}}$ subtracted from unity.

\section{E. Computational Results}

Computational results are tabulated by hour of day to show diurnal variability and by month to show seasonal variability. In addition to the integrated $\mathrm{P}_{\mathrm{CA}}$ results, PACER also computes failure mode probabilities $\left(\mathrm{P}_{\mathrm{FM}}\right)$ for each individual parameter. These can be used to qualitatively assess the relative contribution each parameter constraint makes to the overall climatological availability, with the following caveat: in general, $\mathrm{P}_{\mathrm{V}}$ is not equal to the sum of the individual $\mathrm{P}_{\mathrm{FM}}$ values due to the aforementioned lack of statistical independence between the individual environmental parameters. This is indicated by noting that during a given reporting interval, more than one parameter may fail the respective NECs.

As an example, consider a scenario definition containing the seven parameter constraints listed in Table 2. As shown, the data for the distance to the nearest lightning strike is be measured relative to Launch Complex 39B (LC39B). All other parameter datasets are taken from operational hourly observations made at the KSC Shuttle Landing Facility (SLF). The resulting output table for this scenario is shown in Table 3. In Table 3, values shown in black give probabilities for specific month/hour of day combinations. For example, $77.4 \%$ of all valid observation intervals during hour 0 Coordinated Universal Time (UTC) in the month of January have observations that satisfy all seven specified NECs. Values shown in red give probabilities for specific months regardless of the hour of the day. For example, $70.3 \%$ of all valid observation intervals in January have observations that satisfy all NECs. Values shown in blue give probabilities for specific hours of the day regardless of the month. For example, $81.0 \%$ of all valid observation intervals during hour 0 UTC have observations that satisfy all NECs. Finally, the value shown in green gives the net probability for all valid observation intervals regardless of month or hour of the day. In this example, $79.0 \%$ of all valid observation intervals have observations that satisfy all specified NECs.

Table 2. List of parameter constraints for an example scenario definition.

\begin{tabular}{|c|c|c|}
\hline CONSTRAINT & NEC & SITE \\
\hline 1 & Lightning Distance $>18.5 \mathrm{~km}$ & $\mathrm{LC}-39 \mathrm{~B}$ \\
2 & Peak Wind Speed $<15.8 \mathrm{~m} / \mathrm{s}$ & $\mathrm{KSC} / \mathrm{SLF}$ \\
3 & Minimum Temperature $>0.6^{\circ} \mathrm{C}$ & $\mathrm{KSC} / \mathrm{SLF}$ \\
4 & Maximum Temperature $<37.2^{\circ} \mathrm{C}$ & $\mathrm{KSC} / \mathrm{SLF}$ \\
5 & Cloud Ceiling Height $>2.5 \mathrm{~km}$ & $\mathrm{KSC} / \mathrm{SLF}$ \\
6 & Visibility $>8.0 \mathrm{~km}$ & $\mathrm{KSC} / \mathrm{SLF}$ \\
7 & No Thunderstorms Reported Nearby & $\mathrm{KSC} / \mathrm{SLF}$ \\
\hline
\end{tabular}

4

American Institute of Aeronautics and Astronautics 
Table 3. Example climatological probabilities (\%) of satisfying all NECs.

\begin{tabular}{|c|c|c|c|c|c|c|c|c|c|c|c|c|c|}
\hline Hour (UTC) & Jan & Feb & Mar & Apr & May & Jun & Jul & Aug & Sep & Oct & Nov & Dec & All months \\
\hline 0 & 77.4 & 74.7 & 79.5 & 88.2 & 85.0 & 81.5 & 86.6 & 83.3 & 80.9 & 82.0 & 77.4 & 75.4 & 81.0 \\
\hline 1 & 78.2 & 76.4 & 83.3 & 86.8 & 87.5 & 85.1 & 89.0 & 86.6 & 84.9 & 81.8 & 80.5 & 74.5 & 82.9 \\
\hline 2 & 76.0 & 74.8 & 84.8 & 88.7 & 88.8 & 85.1 & 92.2 & 88.4 & 84.1 & 83.2 & 79.3 & 72.3 & 83.2 \\
\hline 3 & 75.0 & 73.3 & 83.3 & 88.4 & 90.4 & 90.1 & 94.7 & 92.7 & 89.1 & 84.4 & 78.6 & 70.5 & 84.2 \\
\hline 4 & 74.7 & 76.5 & 81.9 & 89.4 & 91.9 & 92.0 & 95.7 & 92.1 & 88.4 & 85.9 & 77.4 & 71.7 & 84.8 \\
\hline 5 & 71.9 & 76.1 & 78.7 & 89.5 & 89.8 & 93.7 & 96.4 & 95.4 & 90.2 & 83.5 & 77.2 & 70.4 & 84.3 \\
\hline 6 & 68.6 & 74.2 & 81.0 & 89.5 & 89.7 & 93.8 & 96.2 & 94.8 & 93.0 & 84.3 & 75.2 & 67.7 & 84.0 \\
\hline 7 & 69.5 & 71.5 & 81.1 & 86.7 & 89.1 & 93.3 & 95.2 & 95.6 & 91.1 & 83.5 & 72.8 & 67.7 & 83.1 \\
\hline 8 & 67.3 & 71.9 & 79.3 & 86.1 & 88.4 & 91.5 & 95.2 & 95.6 & 90.4 & 83.5 & 72.7 & 65.9 & 82.3 \\
\hline 9 & 65.2 & 67.2 & 78.0 & 84.0 & 88.2 & 89.6 & 93.6 & 94.9 & 90.6 & 81.2 & 72.1 & 67.4 & 81.0 \\
\hline 10 & 63.9 & 67.5 & 75.5 & 80.1 & 85.0 & 85.3 & 92.6 & 94.4 & 87.4 & 79.4 & 71.4 & 63.4 & 78.8 \\
\hline 11 & 59.5 & 61.2 & 73.3 & 75.8 & 77.3 & 79.5 & 86.7 & 86.8 & 81.6 & 74.6 & 69.3 & 61.0 & 73.9 \\
\hline 12 & 58.7 & 58.4 & 67.5 & 78.2 & 82.3 & 83.5 & 88.1 & 89.1 & 82.4 & 70.1 & 64.3 & 56.3 & 73.3 \\
\hline 13 & 57.7 & 59.4 & 72.9 & 80.2 & 88.3 & 86.3 & 91.2 & 91.7 & 84.3 & 75.6 & 67.4 & 57.4 & 76.0 \\
\hline 14 & 66.2 & 65.8 & 74.8 & 80.7 & 83.9 & 84.5 & 90.9 & 93.4 & 84.5 & 79.2 & 70.5 & 65.7 & 78.4 \\
\hline 15 & 70.7 & 68.0 & 73.1 & 81.5 & 84.5 & 80.4 & 90.9 & 88.7 & 82.4 & 78.3 & 71.0 & 70.4 & 78.4 \\
\hline 16 & 72.9 & 68.1 & 75.8 & 79.3 & 84.2 & 77.4 & 85.6 & 82.8 & 79.7 & 80.1 & 71.9 & 69.4 & 77.3 \\
\hline 17 & 71.8 & 69.0 & 76.6 & 78.7 & 86.0 & 73.9 & 79.5 & 77.3 & 76.5 & 77.5 & 72.4 & 70.5 & 75.8 \\
\hline 18 & 72.1 & 73.5 & 78.8 & 80.7 & 84.8 & 69.4 & 73.1 & 68.7 & 76.6 & 77.9 & 72.2 & 70.0 & 74.9 \\
\hline 19 & 73.2 & 72.6 & 77.4 & 82.3 & 83.8 & 65.1 & 68.6 & 69.3 & 76.4 & 76.3 & 73.2 & 70.1 & 74.0 \\
\hline 20 & 72.6 & 71.7 & 78.6 & 84.1 & 82.8 & 65.3 & 68.5 & 68.9 & 75.6 & 77.6 & 73.6 & 69.3 & 74.1 \\
\hline 21 & 73.7 & 72.2 & 76.7 & 84.3 & 84.3 & 70.2 & 71.3 & 72.8 & 77.2 & 77.1 & 73.7 & 69.4 & 75.3 \\
\hline 22 & 75.3 & 73.7 & 78.6 & 85.7 & 84.6 & 74.1 & 74.4 & 77.9 & 78.6 & 77.8 & 73.8 & 71.5 & 77.2 \\
\hline 23 & 74.6 & 73.4 & 80.7 & 84.7 & 86.7 & 77.6 & 80.9 & 78.3 & 80.0 & 79.9 & 75.1 & 72.7 & 78.7 \\
\hline All hours & 70.3 & 70.5 & 78.0 & 83.9 & 86.1 & 82.0 & 86.6 & 85.8 & 83.6 & 79.8 & 73.5 & 68.4 & 79.0 \\
\hline
\end{tabular}

\section{Natural Environments Datasets}

The datasets used within PACER to compute the climate analyses outputs represent conditions at the launch site, abort landing site(s), and the nominal landing site. KSC contains an extensive, dense network of instrumentation used to characterize the local meteorological environment. For sea states, available in-situ measurements and satellite-based archives are inadequate to efficiently compute launch availabilities. Therefore, global reanalysis datasets are used to assess the oceanic environment applicable to vehicle landing and recovery ${ }^{2}$. Outputs from these datasets define probabilities of meeting user-specified constraints based on the climatology of the regions of interest.

\section{A. Surface Meteorological Environments}

Archived data from the SLF define the surface meteorological environment at KSC. The SLF archive consists of hourly data records over the 1978-2007 POR. Parameters include mean wind speed and wind direction, peak wind speed, ceiling height, visibility, temperature, dewpoint temperature, atmospheric pressure, precipitation, thunderstorms, visibility, and sky cover. The instrumentation measures pressure, temperature and relative humidity at 2 meter $(\mathrm{m})$ altitude, and wind speed and direction at $10 \mathrm{~m}$ altitude. Air pressure is reported relative to mean sea level. Trained observers provide reports of precipitation and thunderstorm occurrences as well as estimates of visibility, ceiling height, and sky cover. Figure 1 shows a sample time series plot of meridional and zonal wind components, temperature, and dewpoint from the SLF. 


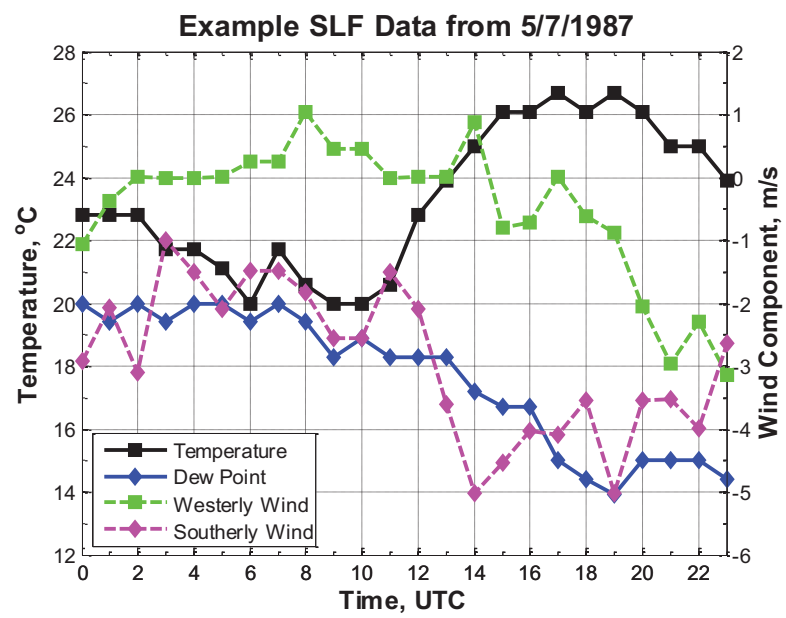

Figure 1. Example time series plot of SLF mean wind component velocities, air temperature, and dewpoint temperature.

\section{B. Lightning Observations}

The National Lightning Detection Network (NLDN) ${ }^{3}$ dataset provides surface lightning information for KSC. The NLDN consists of over 100 ground-based sensors spaced around the country which measure electromagnetic discharges from cloud-to-ground lightning strikes. A central processor records signal receive time and direction from all sensors detecting a given strike and interpolates the actual strike time and location. The NLDN includes sensors located throughout the state of Florida, including several within observing distance of KSC. The NLDN archive within PACER contains the timestamp and location of every cloud-to-ground lightning occurrence within $50 \mathrm{~km}$ of LC-39B over the 1997-2013 POR.

\section{Sea States}

Output from the European Centre for Medium Range Weather Forecasts Re-Analysis (ERA) dataset is used to quantify PACER's sea state assessment. Traditionally, the 40-year ERA dataset (ERA-40) has been used as it consisted of the "longest and most complete wave dataset" parameters including significant wave height, average wave period, and $10-\mathrm{m}$ wind speed on a global $1.5^{\circ}$ longitude by $1.5^{\circ}$ latitude grid. Outputs exist every six hours over the 1957-2001 POR, but PACER incorporates data from 1971-2001 to comply with World Meteorological Organization standards and to make use of satellite data assimilation. The new Interim ERA (ERA-I) $)^{5}$ dataset will soon replace the ERA-40 dataset because the ERA-40 dataset has not been updated since 2001. Figure 2 shows an example output from the ERA-I. The ERA-I dataset

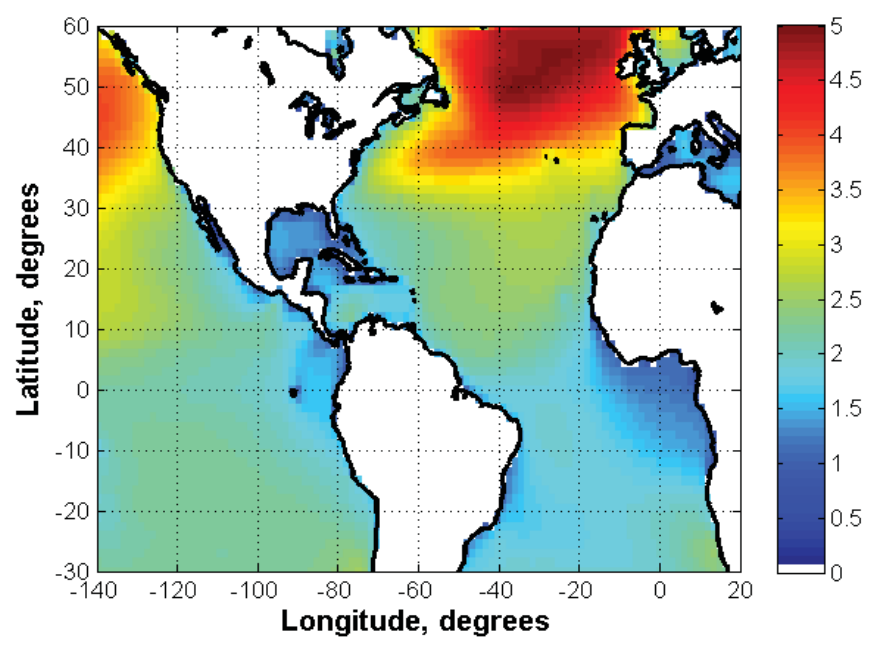

Figure 2. Map of ERA-I mean significant wave height at each gridpoint during January over the 1979-2012 POR .

6

American Institute of Aeronautics and Astronautics 
contains the same parameters of interest on the same global grid as the ERA-40 dataset, but with an updated 19792012 POR. Moreover, the ERA-I dataset contains wind reports every three hours instead of every six hours.

\section{ESD Program Climate Analysis Needs}

With the new launch system SLS and crew vehicle Orion, NASA is looking to send human and scientific missions beyond low Earth orbit (LEO). Specific Design Reference Missions (DRMs) are still being defined, but NASA's goal is to have the capability to support a number of DRMs, including lunar fly-by and multi-launch Mars missions. To be able to perform these missions, the integrated vehicle must be able to launch on schedule. This is especially critical when those DRMs require multiple launches or have a narrow launch window. Therefore, having a system that is robust enough to have high launch availability regardless of weather conditions is highly desirable, and understanding what environments most affect that capability is critical. Table 4 lists the current launch weather and range safety element parameters that are being utilized within PACER for ESD Program launch climate analyses.

Table 4. List of environmental launch parameters
defined within PACER for the ESD Program.
\begin{tabular}{|c|}
\hline LAUNCH WEATHER ELEMENTS \\
\hline Air Temperature \\
Land-based Peak Wind Speed \\
Presence of Precipitation \\
Presence of Thunderstorms \\
Oceanic Mean Wind Speed \\
Significant Wave Height \\
Average Wave Period \\
\hline RANGE SAFETY ELEMENTS \\
\hline Cloud Ceiling Height \\
Visibility \\
Distance to Nearest Lightning Strike \\
\hline
\end{tabular}

\section{A. Launch Vehicle Climate Analysis}

The SLS launch vehicle is responsible for safely delivering NASA crew vehicles and payloads to desired orbits. To accomplish this task, the SLS launch vehicle must meet its functional and performance requirements during and after exposure to the natural environments. The preferred method to mitigate the effects of these natural environments is through robust design of the vehicle. However, it is understood that a robust design is not always attainable for some of the natural environments due to schedule/cost impacts or structural limitations. For these cases, operational controls are employed to mitigate the effects on the launch vehicle. For example, designing the launch vehicle to withstand a direct lightning strike can have a significant cost impact. Therefore, operational controls are implemented to minimize exposure to lightning.

The set of natural environments used for design are chosen in order to provide high launch availability. The adverse effect of operational controls is that they reduce launch availability, since the launch vehicle cannot be certified to operate in those environments. For the SLS Program, launch availability due to natural environments is managed via a TPM. Trade studies are conducted to compare the impact of operational controls on launch availability to the feasibility of a robust vehicle design. The natural environments used to determine launch availability include the surface observations in Table 4 excluding the ocean/sea environments since the SLS launch vehicle is not required to operate in those environments. Upper-air winds are also included in the SLS launch availability assessment, since high winds and large wind shears can induce vehicle loads that could compromise the structural integrity of the vehicle. Constraints for the applicable environments in Table 4 are evaluated by PACER to determine the launch availability relative to surface observations. Upper-air winds are evaluated through Monte Carlo simulations using random wind profiles. The simulation outputs are compared to pre-determined trajectory 
and load constraints to determine availability due to upper-air winds. These results are combined with the PACER results to determine the overall SLS launch availability, which are then reported in the TPM. The SLS Program management team uses cost/schedule impacts and the TPM data to decide between designing a vehicle to withstand the effects of the natural environments or implementing operational controls to limit exposure to the natural environments.

\section{B. Crew Vehicle Climate Analysis}

The Orion crew vehicle is responsible for safely transporting astronauts on exploration and scientific missions and returning them to Earth. The points of destination will be determined as NASA further defines its DRMs, but the target launch and landing zones are already being discussed (Figure 3) and the environments in those regions are being assessed for potential Orion launch and landing availabilities.

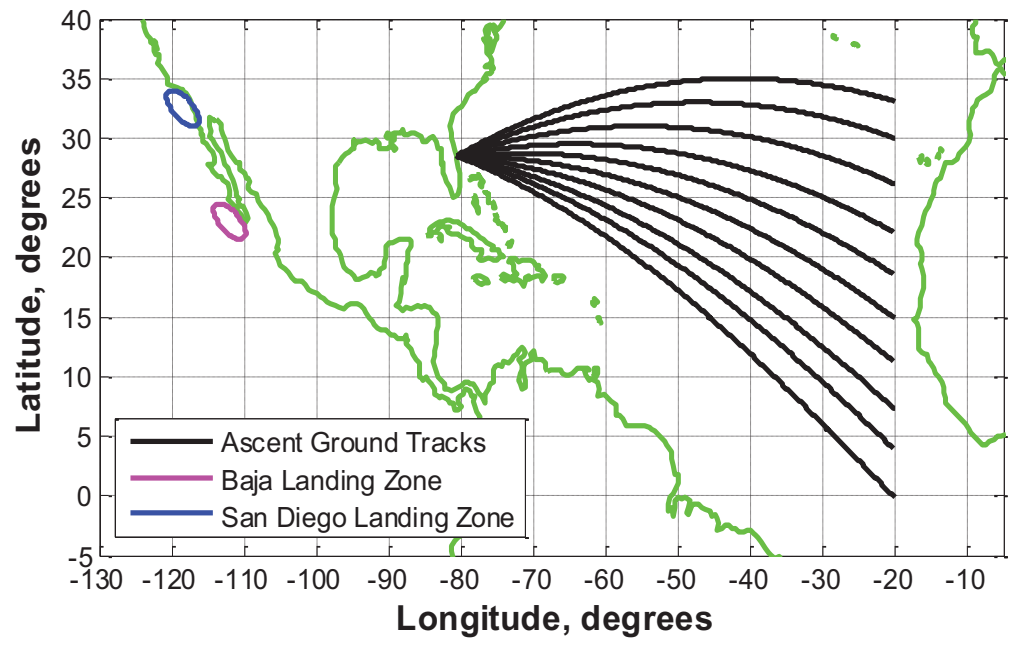

Figure 3. Preliminary ascent tracks and landing zones for natural environments and sea state assessments for the Orion crew vehicle.

Orion must be able to abort and safely land in the water anywhere along the launch vehicle ascent trajectory, including on-pad and near-pad aborts that would place the vehicle just offshore from KSC. Should an anomaly occur during in-space pre-orbital ascent that would prevent Orion from reaching its intended orbit/mission, Orion would then need to perform an AOA maneuver and land in the Pacific Ocean. Currently, the targeted AOA landing zone is just offshore from the Baja Peninsula (see Figure 3). Orion must also have the capability to perform nominal landings at a pre-determined landing site. During CxP, PACER climate analyses were performed to look at the potential of both land and water nominal landings at various locations. For land landing zones, data from identified sites were used by PACER to perform the land landing climate analysis. The datasets for those landing sites were similar to that of the SLF dataset. The sea states were evaluated using the ERA-40 dataset. Eventually, it was determined during CxP that Orion would nominally land in water as opposed to on land, and Orion is continuing to design for this method under the ESD Program.

The Orion vehicle consists of three modules - the Launch Abort System (LAS), the Crew Module (CM), and the Service Module (SM). The LAS consists of a solid rocket-powered launch motor that sits atop the CM. This system can pull Orion away from the launch vehicle should a catastrophic anomaly occur on the launch pad or during ascent. While on the pad, the LAS would pull Orion off the integrated stack and steer it out over the Atlantic Ocean. Once it is a safe distance away, parachutes will be deployed and Orion will safely land the crew offshore from KSC. The CM is the crew capsule in which the astronauts reside. It is similar in shape to the Apollo capsules but much larger in size. The SM contains the propulsion system that allows Orion to fly beyond LEO. The SM is disposed of during re-entry.

To perform a launch climate analysis for Orion, PACER is used to evaluate all of the launch weather parameters listed in Table 4. The range safety elements can be assessed if requested, but those parameters are usually folded in when the integrated vehicle launch climate analysis is performed. There are a different set of sea state criteria for abort/off-nominal water landings versus nominal water landings. For launch climate analyses, the abort/off-nominal criteria are used for evaluation in PACER. The sea states are analyzed across the varying potential ascent trajectory 
ground tracks plus the AOA zone. The angle of impact of the $\mathrm{CM}$ as it hits the water/waves affects the loads the crew will incur upon splashdown. Protecting for these sea states can affect crew survivability, but it can also affect launch availability throughout the year. The wind and wave conditions in the Atlantic and Pacific Oceans vary over the seasons. By using PACER to perform a climate analysis, results can show trends - such as seasonal trends - in which the sea state criteria are exceeded. That information can be used in risk assessments to understand crew risk as well as cost and schedule impacts. Upper-air winds must be evaluated through Monte Carlo simulations using wind profiles to assess the response of the LAS and parachute system to the wind environment. These results can be combined with the PACER results to understand the overall Orion launch availability.

To perform a landing climate analysis for Orion, PACER is used to evaluate only the sea state parameters listed in Table 4 for nominal landing constraints instead of launch constraints. Currently, the nominal landing zone defined for Orion is off the coast of San Diego, California. Should the location of that zone change, PACER can be updated to perform a nominal landing climate analysis for any given water landing zone determined by the MPCV Program. Results from upper air assessments from Monte Carlo simulations using random wind profiles for the nominal water landing zone can be combined with the PACER results to understand the overall Orion landing availability.

Prior to operational missions launching atop the SLS vehicle, Orion is currently on schedule to launch EFT-1 in late 2014. This is a short-duration (4 hours), two orbit mission that will see Orion launched aboard a Delta IV Heavy Lift launch vehicle. After separation, Orion will perform one low apogee orbit before it swings out to a 3600-mile, high apogee orbit (approximately 15 times higher than the current orbit of the International Space Station). Orion will then perform a direct entry and land in waters off the coast of the Baja Peninsula - near the same water landing zone it would target for an AOA landing. Because EFT-1 is a short mission, both the launch and landing criteria must be met before a commit to launch. PACER is being used to perform a climate analysis for this unique situation by capturing the off-nominal sea states along the ascent trajectory and the nominal sea states within the Baja landing zone.

\section{Integrated Vehicle Climate Analysis}

The overall, integrated vehicle launch availability is being formally captured in an ESD LA TPM ${ }^{6}$. The ESD LA TPM takes into consideration the reliability of the overall system (including ground system operations and the natural environments), the likelihood of a failure, and the time required to recover from an anomaly. The MSFC Natural Environments Branch provides both monthly and annual results for the ESD LA TPM. For SLS cargo-only missions, a PACER launch climate analysis is performed for all the parameters listed in Table 4 excluding sea state criteria. For SLS-Orion missions, all parameters in Table 4 - including the range safety elements - are assessed within PACER.

PACER is only used to analyze surface environmental parameters by checking climatological data against specified criteria thresholds. The model does not consider dynamic vehicle responses to given environments. As such, aloft winds are not assessed in PACER because it is not possible to define a specific constraint without doing a high-fidelity trajectory simulation incorporating a detailed vehicle configuration. It should also be noted that, in relation to sea surface conditions at abort landing zones, the climatology of hurricane conditions are included in the reanalysis dataset used by PACER. Other analyses of historical hurricane tracks in the Atlantic and Pacific oceans, including land-falling hurricanes near the launch and/or landing sites, could provide important information for mission planning and operations development activities, but should not be explicitly included in ESD LA TPM calculations due to the possibility of adding undue conservatism and artificially lowering the computed availabilities.

\section{Conclusion}

Launch and landing availabilities require integrated analyses with relation to natural environments. These environments drive impacts to the vehicles being designed, as well as crew risk, cost, and schedule impacts. The capability of the MSFC Natural Environments Branch to perform these types of analyses for varying locations and scenarios allows the Programs to assess the risks and form mitigation strategies early on. SLS and Orion engineering design is ongoing. As vehicle specifications evolve and DRM details mature into actual mission plans, it is expected that new scenarios, including updated parameter thresholds and NEC definitions, may need to be analyzed in relation to launch and landing availability. The MSFC Natural Environments Branch maintains a large inventory of statistical analysis tools, environment definition models, and observational data archives. These assets provide the capability to support many different hardware design and operational planning analyses. Expertise is available for both the application of current models and datasets and in the development of new models and datasets. Delivered 
products can support a wide variety of engineering analyses and provide decision tools for design engineers and mission planners throughout the lifecycle from concept to design and throughout operations.

\section{References}

${ }^{1}$ Burns, K. L., "Probabilities of Atmospheric Conditions and Environmental Risk: Design and Operation of a New Analysis Tool for Computing Launch and Landing Availability With Respect To Weather and Natural Environmental Conditions", ESTSG-FY11-536, 20 Apr. 2011.

${ }^{2}$ Barbré, R.E., and Keller, V.W., "Sea State and Weather Assessment Capability for NASA's Constellation Program," $13^{\text {th }}$ Conference on Aviation, Range, and Aerospace Meteorology, P2.2, American Meteorological Society, New Orleans, LA., 2008.

${ }^{3}$ Orville, R.E., "Development of the National Lightning Detection Network," Bulletin of the American Meteorological Society, Vol. 89, No. 2, 2008, pp. 180-190.

${ }^{4}$ Caires, S., and Sterl, A., "A New Nonparametric Method to Correct Model Data: Application to Significant Wave Height from the ERA-40 Re-Analysis," J. Atmos. Oceanic Technol., Vol. 22, 2004, pp. 443-459.

${ }^{5}$ Dee, D.P, Uppala, S. M., Simmons, A. J., Berrisford, P., Poli, P., Kobayashi, S., Andrae, U., Balmaseda, M. A., Balsamo, G., Bauer, P., Bechtold, P., Beljaars, A. C. M., van de Berg, L., Bidlot, J., Bormann, N., Delsol, C., Dragani, R., Fuentes, M., Geer, A. J., Haimberger, L., Healy, S. B., Hersbach, H., Hólm, E. V., Isaksen, L., Kallberg, P., Köhler, M., Matricardi, M., McNally, A. P., Monge-Sanz, B. M., Morcrette, J.-J., Park, B.-K., Peubey, C., de Rosnay, P., Tavolato, C., Thépaut, J.-N., and Vitart, F, "The ERA-Interim Reanalysis: Configuration and Performance of the Data Assimilation System," Q. J. R. Meteorol. Soc. Vol. 137, 2011, pp. 553-597.

${ }^{6}$ Watson, M., Staton, E., Cates, G., Finn, R., Altino, K.M., Burns, K.L. "Use of DES Modeling for Determining Launch Availability for SLS," SpaceOps2014 13th International Conference on Space Operations, AIAA, Pasadena, CA (submitted for publication) 